\title{
Research on Universities Digital Education Resource Sharing Mechanism
}

\author{
Bing Liu ${ }^{1, a}$ \\ ${ }^{1}$ Institute of NBC Defense, Beijing, China, 102205 \\ a email
}

Keywords: Universities, Digital Education, Resource Sharing Mechanism

\begin{abstract}
In the 21st century, China has entered a new era of changing higher education from elite education to mass education and universities have increasingly become the gathering center in the region a lot of talent and resources, as a regional rapid and coordinated development of the main driving force and source. However, due to social conflicts unlimited demand for excellent education resources and the limited supply of excellent education resources between the increasingly prominent, in this context, to promote open sharing of educational resources to deepen the strategic choice of higher education for sustainable development.
\end{abstract}

\section{Introduction}

In the 21st century, Chinese higher education received an unprecedented rapid development, access by the elite education to mass education in the new era of change. Universities are also increasingly becoming the center of the region gathered a lot of talent and resources, become the main source of power and promote regional rapid and coordinated development. People contradictions and educational resources for the needs of the insufficient supply of educational resources highlights. Former Minister of Education Zhou Ji pointed out: the shortage of high-quality teaching resources is a long-standing problem of higher education, developed countries. In this regard, just to play our institutional superiority, concentrate on building, widely shared. In this context, promoting open sharing of educational resources has become a strategic choice to deepen the Higher Education for sustainable development. In the country's vigorous advocacy and the promotion of various projects in recent years, higher education has made the sharing of resources building a great achievement, but due to technical reasons, demand, funding, management, and many other aspects of the system, resulting in between Universities digital educational resources to poor share performance, with people sharing practical results originally envisaged, there are still large gaps, needs improving.

Progress and development of modern computing technology and network resource sharing higher education provides the means to achieve. Since 2009, the cloud computing more and more people are familiar with, is the current hot topic, it has been widespread concern. Cloud computing is regarded as the next revolution in the scientific community, it is not only technology, but also represent a service concept and service models. It will make the business model work and fundamental change. The sudden emergence of cloud computing, had a profound impact on all sectors, whether it is the IT industry's leading manufacturers, research institutes, or ordinary users, are cloud computing devoted a great deal of enthusiasm. Cloud computing technology with its powerful features already in some areas and industry has been a preliminary application.

\section{Digital Education Resources and Its Classification}

Digital Education Resources refers to all at this stage or in the future can be digitized information school education services, school resources and external resources into two parts. External resources refers to resources on the Internet and on CD-ROM, campus resources include: school everyday documents, such as various documents, school calendar, notices, newsletters; teachers, students basic information file; daily teaching information, timetable, transfer courses notifications, open class ; questions, exercises, exams, term reviews; teacher preparation notes, electronic lesson plans, papers; courseware, digital audio-visual materials; disciplinary thematic information; 
electronic books; all kinds of materials and other logistics management. Compared with the information environment, digital educational resources have a more direct role in education applications.

\section{Economic Characteristics of Digital Education Resources}

Does Not Fully Competitive. Consumption of digital educational resources is in line with the characteristics of public goods. Within a certain range, with a multi-user educational resources can be used, and will not provide the additional use of a person to bring it to reduce the effectiveness of other people, not because others are leaving to enjoy digital educational resources the total reduction, but because others enjoy the original person whose interests or provider is compromised, resulting in devaluation of digital educational resources for their owners.

The Marginal Cost Is Very Low. Marginal in economics generally indicate an increase in this sense, the marginal cost is the change for each additional unit of production caused by the cost. Digital educational resources with high production costs and the first draft of a low marginal cost, that is the first time in its production processes requires higher costs, and after only a small amount of maintenance costs can be reused many times. Increasing the marginal cost of a digital educational resources provided by the user to bring in a range of not even be ignored, so that any consumer can count on a ride. Phenomenon is very difficult.

Exclusive and Non-Exclusive. While the digital educational resource itself is non-exclusive, but in the sharing process, but because of the owners' interests, while making some digital educational resources (such as those involving intellectual property issues) to pay a fee before they enjoy, the digital educational resources have exclusive. Digital educational resources for the performance of non-exclusive, the same resource can be used simultaneously by different users, that resource will not be used and devalued.

The Spatial Distribution Is Uneven. Digital education resources uneven regional distribution, not only from the cultural and historical differences accumulated precipitation over the country, but also with the local economic level and university level have a great relationship. In general, the economy, culture, scientific research, education, the more developed areas, types of colleges and universities, the higher the educational level, with richer digital educational resources accordingly, on the contrary, the more the digital educational resources rich areas, research, culture, economy and education are also more developed, is a mutually reinforcing relationship between the two. In central and western China and some of the region's economy is relatively underdeveloped, lagging behind by these three digital educational resources are relatively scarce, therefore, to establish an effective digital education resources sharing mechanism is conducive to promoting the balanced development of our country.

Scarcity. Despite the large amount of digital educational resources, but for the user at a specific time, specific needs of a particular space, the effective digital educational resources are always limited. On the one hand, the number of useful educational resources shall be obtained from a large number of complex information, but due to the development costs of digital educational resources and timely decision-making time limit restrictions, people master the digital educational resources is not always completely equal. On the other hand, although digital educational resources can provide for people to use, but from a practical point of view, a useful digital educational resources, but because of someone else's use and indirectly reduce the value of the user.

\section{The Significance of University Digital Education Resources Sharing}

It is Conducive to Narrow the Resources Gap between the Rich and the Poor. Due to economic development, education, history and other aspects of digital educational resources between universities there are obvious gaps between rich and poor, a lot of resources on a larger scale, of the older universities in major cities, which uneven distribution of resources, if not effectively improved, can easily result in a lot of duplication of resources, is not conducive to the balanced development of scientific research and education. To bridge this gap, we first need the resources are 
relatively barren areas and universities appropriately increase investment in order to improve the current behind the resources and the environment. However, this investment, after all, only a limited, excessive capital investment is not only unrealistic but also likely to cause a lot of waste. Promoting digital educational resource sharing among universities is an effective way to solve the problem, it will enable the institutions had fewer resources at a lower cost to obtain higher returns, minimizing the gap between resource-rich and universities, to achieve balanced development .

Help to Improve the Utilization of Digital Educational Resources. Currently, redundant construction serious university resources, resources can not maximize its effectiveness, many valuable resources are shelved, there are nobody cares, causing great waste. By Digital Education Resources Sharing in University, said the phenomenon can be well improved. First, the construction of resource sharing can be more systematic. Through scientific planning, resource duplication and a lot of "information island" phenomenon can be avoided to the greatest extent, while the various colleges and universities can put more money into the resource library in the school building has professional features, which not only reduces the waste resource more so that the number of high-quality resources to be increased significantly. Secondly, through the sharing of digital educational resources Universities can have a larger user base, not only for the more conventional resources can be used by the user, and some resources in the school-utilized can also maximize their effectiveness, make use of resources rate has been greatly improved.

Help to Meet the Resources Needs of Diverse User. With the continuous development of society, university research continues to improve the level of education, university user demand for resources is also constantly updated, the urgent need for comprehensive, diverse forms of scientific, innovative and complete digital educational resources, and this demand alone school the resources are often unable to meet, only to realize effective sharing of digital educational resources between different universities, other colleges and universities as a resource to complement and extend the school's resources in order to provide a higher quality of service for university users to meet their increasingly diverse digital Education resource Requirements

\section{University Digital Education Resource Sharing Mechanism}

Organization and Coordination Mechanism. In the cloud-based Digital Education Resource Sharing level can be established through resource sharing cloud management committee coordination mechanisms. Resource sharing cloud management committee is a coordinating body for the university resource sharing and the main function is to coordinate the interests of the relationship between colleges and universities, to ensure the sharing of resources to achieve. Issues, such as who bears how much of the digital sharing of educational resources construction task, how the distribution of benefits and shared resource charges. Member of the management Board may by the College is responsible for the implementation of resource sharing leadership and the local education authority composed. shared cloud management Committee play a leading role in coordinating the interests of all parties to integrate resources, played the role of arbitration to solve the problem due to the sharing of resources in the process, the university is easy from the perspective of intellectual property protection or parochialism common Thinking their own interests, which will affect the cooperation between the sharing of resources and processes inter-school through voluntary principles of fairness and independent consultation, the sharing of resources needed cloud management committee to develop mutually acceptable agreement on sharing and rules.

Integrated Unified Mechanism. As a long time building its own system resources, fragmented, resulting in the presence of a large number of heterogeneous resources. Cloud computing digital education resources sharing environment, integration of resources is necessary. First, in a cloud computing environment requires local data resources to organize the package later, you can upload to the cloud, to facilitate management and sharing of digital educational resources cloud sharing mode requires the establishment of a unified basic information for the realization of digital educational integration of resources from different service systems, providing unified authentication services, unified and open API and unified API hosting. According to the actual needs of the school 
digital resource sharing, the resources available to colleges and universities based on the formation of a "logic of centralized, decentralized physical" cloud shared resource Center. these resources physical on can be delivered to a third party cloud operators to save administration can also be stored on the local machine to form a shared virtual resource pool logical by uploading to the cloud you will need to have a common demand resources or resources to gather homogeneous by technical support, unified process, the formation of massive data exchange, manage, share and query, form a unified cloud storage repository. Publish the sharing of resources by focusing on the integration of the same platform, focused on mining to be enhanced digital educational resources utilization.

Security Protection Mechanism. Generally speaking, school digital educational resources available free of charge in addition to the shared portion, also involves a number of issues accounting rules and intellectual property rights, universities these data resources to deliver to a third party cloud services providers in the future, since the digitizing Senate confidentiality of information management resources, universities will lose data control over resources, thereby increasing the security of resources. Resources by the cloud service delivery management, the security issues of digital educational resources mainly from resources transferred over the Internet, how ensure the security and backup issues resources in the cloud server data.

Continuing Incentives Mechanism. The principle of mutual benefit, contribute more, the greater the share rights. Mobilize the people's enthusiasm through incentives to share rights, obligations and contribute resources to match. Points punish violators, those who sit back and enjoy spending integral contributor to the points of principle, by constructing the "Repository points system" to motivate and mobilize the broad participation of users in the repository points system model, and some of the resources through commercialization operation and social reward and punishment mechanism, each user can freely switch between the dual roles of consumers and builders, both can redeem into cash to get real income, cash may be used to recharge the way and forms Rewards to earn points. In this way, both the protection of intellectual property rights play a role, but also to ensure the repository construction quality and efficiency improvement.

\section{Conclusion}

With the rapid development of computer technology, communication technology and its wide range of applications in education, higher education, scientific research and the demand for more and more computer technology, university users to share digital educational resources in increasing demand, but in between Universities digital resource sharing the process of education, there are still many problems and obstacles. This paper combines theories about remote education services, educational technology and economics theory, make an in-depth analysis on the status quo and obstacles of the university digital education resources, and further promote the mechanism of University Digital Resources for Education and stable operation of a more systematic research and discussion.

\section{References}

[1] Kang Ning. Educational Research, Vol. 6 (2014) No 53, p.25-26

[2] Wang Qunyong. China Distance Education, Vol. 12 (2015) No 27, p.74-76

[3] Jing Jianfen, Hou Xu. Educational Research, Vol. 30 (2014) No 19, p.144-145

[4] Wang Kuailiang. Higher Education Research, Vol. 29 (2008) No 27, p.21-23

[5] Zhang Gongxu, Sun Jing. China Distance Education, Vol. 8 (2013) No 27, p.57-60 